\title{
Verfassung und Verfassungsgerichtsbarkeit in Peru nach Fujimori
}

\author{
Von Jürgen Samtleben, Hamburg
}

\section{A. Einleitung}

Die Regierungszeit von Alberto Fujimori Fujimori (1990-2000) in Peru war gekennzeichnet durch eine fortschreitende Erosion der verfassungsmäßigen Institutionen, eine zunehmende Zentralisierung und eine faktische Entmachtung des Verfassungsgerichts. Diese Entwicklung wurde damals in Peru selbst in einer Serie von mutigen Leitartikeln von der angesehenen Rechtszeitschrift „Revista jurídica del Perú“ dokumentiert, bis deren Erscheinen eingestellt wurde. ${ }^{1}$ Nach dem Sturz Fujimoris im November 2000 konnte die Zeitschrift wieder erscheinen und begrüßte den politischen Umschwung mit einem flammenden Editorial. $^{2}$ Tatsächlich wurden die gravierenden Eingriffe in die rechtsstaatliche Ordnung bald rückgängig gemacht, die umstrittene Verfassung von 1993, die Fujimori nach seinem „Selbstputsch“ verkündet hatte, blieb jedoch grundsätzlich in Kraft. Dagegen wurde der rechtliche Rahmen der Verfassungsgerichtsbarkeit auf der Grundlage des früheren Rechts völlig neu kodifiziert. Über diese Entwicklung und den heutigen Stand von Verfassungsrecht und -gerichtsbarkeit in Peru soll der nachfolgende Beitrag einen Überblick geben.

\section{B. Die Verfassung von 1993}

\section{Entstehung und Fortgeltung}

Zwei Jahre nach seinem Amtsantritt hatte Fujimori bei seinem Staatsstreich im April 1992 durch Dekret-Gesetz 25418 den Kongress aufgelöst und die Verfassung von 1979 in wesentlichen Teilen außer Kraft gesetzt. Vorangegangen waren Auseinandersetzungen mit dem von der Opposition dominierten Kongress, der sich nicht ohne weiteres für Fujimoris

Editorial: Reinvidicación del Estado de Derecho, RevJurPerú 46 (1996) Nr. 4 S. 11 f.; Sigifredo Orbegoso Venegas, ¿Liquidando el Estado de Derecho?, RevJurPerú 47 (1997) Nr. 10 S. 47 f.; Editorial: Inestabilidad jurídica en el país, ebd. Nr. 11 S. 9 f.; Jorge Avendaño V., ¿Tenemos Estado de Derecho?, RevJurPerú 48 (1998) Nr. 14 S. 7 ff. Siehe auch den kritischen Überblick bei Raúl Ferrero Costa, Una constitución mediatizada, RevDerCiencPol 57 (2000) Nr. 1 S. 157 ff. 
politisches Programm einspannen ließ. ${ }^{3}$ Unter internationalem Druck sah sich Fujimori veranlasst, eine verfassunggebende Versammlung einzuberufen und den von dieser erarbeiteten Verfassungstext einem Referendum zu unterwerfen. ${ }^{4}$ Da sich weite Teile des politischen Spektrums aus Protest gegen den Staatsstreich nicht an der Wahl zur verfassunggebenden Versammlung beteiligten, wurde die Legitimität der neuen Verfassung in Peru schon vor der Abstimmung bestritten. Gleichwohl wurde sie im Referendum am 31.10.1993 mit einer knappen Mehrheit von 52 zu $48 \%$ angenommen - ohne Zählung der $9 \%$ leeren und ungültigen Stimmzettel! - und am 29.12.1993 verkündet. ${ }^{5}$ Inhaltlich beruht sie großenteils auf dem früheren Verfassungstext, wenngleich dieser wesentlich gekürzt und teilweise unschärfer gestaltet wurde. Im Gegensatz zur Verfassung von 1979, die noch den Gedanken der sozialen Gerechtigkeit betonte (Art. 110), bekennt sich die Verfassung von 1993 ausdrücklich zum Wirtschaftsliberalismus (Art. 58 ff.). Als wichtigste Änderungen gegenüber der früheren Verfassung werden die Abschaffung des Zweikammersystems, die Zulassung der Todesstrafe für Terroristen und die Möglichkeit einer unmittelbaren Wiederwahl des Präsidenten hervorgehoben. ${ }^{6}$

Während Fujimori die folgenden Wahlen von 1995 mit überwältigender Mehrheit für sich entscheiden konnte, stieß die entgegen der Verfassung von 1993 im Jahre 2000 durchgesetzte zweite Wiederwahl auf erbitterten Widerstand, der schließlich in Verbindung mit einem hochpolitischen Bestechungsskandal zu seiner Flucht nach Japan und seinem forcierten Rücktritt im November 2000 führte. ${ }^{7}$ Der Kongress nahm jedoch diesen Rücktritt nicht an, sondern erklärte den Präsidenten mit Beschluss vom 21.11.2000 wegen ,andau-

Kennzeichnend für diesen Widerstand ist etwa das Gesetz 25397 vom Februar 1992, durch das auch die normativen Akte des Präsidenten einer stärkeren parlamentarischen Kontrolle unterworfen werden sollten.

Dekret-Gesetz 25684 vom August 1992. Die verfassunggebende Versammlung erließ als erstes die sog. „Ley Constitucional“ v. 6.1.1993, NormLeg 200 § 036, welche die vorläufige Weitergeltung der Verfassung von 1979 unter den Vorbehalt der vom Präsidenten erlassenen Dekret-Gesetze und ihres eigenen Reglements stellte.

Siehe zu diesen Vorgängen ausführlich Maria McFarland Sánchez-Moreno, When a „Constitution“ is a Constitution: Focus on Peru, N.Y.U.J.Int.L.Pol. 33 (2000/01) 561, 564 ff.; Pedro Planas, La Constitución peruana de 1993 ¿ Es una Constitución de consenso?, An DerConstLatinoam 1999, 557, 559 ff.; ferner Jürgen Saligmann, Die peruanische Verfassung von 1993, VRÜ 28 (1995) $193 \mathrm{f}$. Vgl. dazu Domingo García Belaunde, The new Peruvian Constitution, JöR 43 (1995) 651, 654 ff.; eingehend Saligmann (Fn. 5) 195 ff.; zur früheren Verfassung von 1979 siehe Karl-Peter Sommermann, Verfassungsrecht und Verfassungskontrolle in Peru, JöR 36 (1987) $597 \mathrm{ff}$.

7 Siehe zu dieser Entwicklung Dawn Bennett-Ingold, Latin American Democracy: Flourishing or Floundering?, Ga.J.Int.Comp.L. 28 (1999/2000) 111, 120 ff.; Edgar Carpio Marcos, Constitución y reelección presidencial: el caso peruano, BolMexDerComp. 33 (2000) 447 ff.; McFarland Sánchez-Moreno (Fn. 5), 584 ff. 
ernder moralischer Unfähigkeit" des Amtes enthoben. ${ }^{8}$ Eine vom Übergangspräsidenten Paniagua Corazao eingesetzte Kommission sollte das Schicksal der ,geltenden“, aber makelbehafteten Verfassung von 1993 klären und schlug dazu mehrere Lösungen vor. Nachdem bereits die Wahlen des Jahres 2001 auf der Grundlage der Verfassung von 1993 stattgefunden hatten, ${ }^{10}$ erließ der neugewählte Kongress im Dezember 2001 das Gesetz 27600, worin die Fortgeltung der Verfassung ausdrücklich bekräftigt wird. Zugleich wurde durch dieses Gesetz in einer symbolischen Deklaration die Unterschrift Fujimoris unter der Verfassung ,getilgt“, unter Berufung auf den oben genannte Parlamentsbeschluss vom November 2000, und der Verfassungsausschuss des Kongresses beauftragt, einen Entwurf für eine grundlegende Reform der Verfassung unter Beteiligung der Zivilgesellschaft auf der Grundlage der historischen Verfassungstradition und insbesondere der Verfassung von 1979 zu erarbeiten. Bereits im April 2002 wurde von der dafür eingesetzten Expertenkommission ${ }^{11}$ ein entsprechender Entwurf vorgelegt und nach eingehender Diskussion auf nationaler Ebene im Juli 2002 vom Verfassungsausschuss des Kongresses endgültig gebilligt. ${ }^{12}$ Die anschließende Diskussion im Parlament führte jedoch zu keinem Ergebnis, auch eine entsprechende Initiative des Präsidenten Alejandro Toledo blieb letztlich ohne Erfolg. 13

Maßgebend dafür war wohl die Einsicht, dass das Unbehagen an der Verfassung von 1993 sich weniger auf ihren Inhalt als auf die Art ihres Zustandekommens bezog. Dieses Odium glaubte man offenbar durch die „Tilgung“ der Unterschrift von Fujimori ausgeräumt zu haben. Wichtige politische Vorhaben wie die unter Fujimori gestoppte Regionalisierung

Resolución Legislativa 009-2000-CR, NormLeg 294 § 153. Die Rechtsgrundlage dafür ergibt sich aus Art. 113 Nr. 2, das Erfordernis der Annahme des Rücktritts aus Art. 113 Nr. 3 Verf. 1993.

Siehe D.S. 018-2001-JUS v. 25.5.2001, NormLeg $300 \S 186$. Der Vorschlag der Kommission enthielt drei Alternativen: 1) Nichtigerklärung der Verfassung von 1993 und Rückkehr zur Verfassung von 1979 mit entsprechenden Anpassungen, 2) Totalreform der Verfassung von 1993 nach deren Regeln mit der Ziel dem Wiederherstellung der Verfassung von 1979, 3) Abhaltung eines Referendums zur Entscheidung zwischen der Verfassung von 1979 oder Ausarbeitung einer neuen Verfassung. Näher dazu Enrique Bernales Ballesteros, Los caminos de la reforma constitucional en el Perú, AnDerConstLatinoam 11 (2005) 157, 158 ff.

Jedoch wurde zuvor die Möglichkeit der unmittelbaren Wiederwahl des Präsidenten beseitigt (Gesetz 27365 v. 4.11.2000) und das Wahlgesetz von 1997 in verschiedenen Punkten geändert (Gesetz 27387 v. 28.12.2000); zu weiteren Änderungen siehe die aktuelle Übersicht in NormLeg 358 (2006) II $347 \mathrm{ff}$.

Siehe D.S. 004-2002-JUS v. 1.2.2002, NormLeg 309 § 019.

12

Näher dazu César Landa, The Constitutional Reform in Peru as a stage of Constitutional State, JöR 52 (2004) 87 ff.; span. Fassung in RevJurPerú 54 (2004) Nr. 58 S. 7 ff.; zu dem Verfassungsentwurf auch Carmen Meza Ingar, Reforma de la Constitución Peruana, RevDerCiencPol 59 (2002) Nr. 1 S. 157 ff.; kritisch Omar Alberto Sar, El actual proceso de reforma de la Constitución, RevJurPerú 53 (2003) Nr. 46 S. 149, 159 ff., 163 ff. 
des Landes konnten auch im Wege der Verfassungsänderung vorangetrieben werden. ${ }^{14}$ Vor allem erwies es sich, dass nicht so sehr die Verfassung von 1993 als vielmehr deren Manipulation durch rechtsstaatlich bedenkliche Gesetze die autoritäre Amtsführung Fujimoris ermöglicht hatte. ${ }^{15}$ Dies betraf insbesondere die in der Verfassung garantierte Unabhängigkeit der Justiz, die unter Fujimori einem starken Einfluss der Exekutive ausgesetzt war. ${ }^{16}$ Die zu diesem Zweck geschaffenen Leitungsorgane wurden unmittelbar nach dem politischen Umschwung aufgelöst und die unter Fujimori ergangenen Justizgesetze aufgehoben. ${ }^{17}$ Ähnlich verlief die Entwicklung im Bereich der Verfassungsgerichtsbarkeit, über die weiter unten zu berichten sein wird (siehe unter C).

\section{Verfassung oder ,Dokument" ?}

Für diejenigen, die dem Verfassungstext von 1993 jede Legitimität absprachen und ihn deshalb auch nur als „Dokument“ qualifizieren wollten, war ein solcher Zustand freilich nicht hinnehmbar. Bereits 2002 formierte sich unter der Führung der „Fuerza Democrática“ eine Bürgerinitiative zur Wiederherstellung der Verfassung von 1979. Das Recht der Bürgerinitiative war - entsprechend dem plebiszitären Charakter der von Fujimori verkörperten „democradura“ - erstmals in Art. 107 der Verfassung von 1993 verankert und durch Gesetz 26300 von 1994 geregelt worden. Allerdings war dieses Gesetz in der Folge unter dem Eindruck eines gegen Fujimori geplanten Referendums durch weitere gesetzgeberische Maßnahmen seiner praktischen Wirkung beraubt und erst nach dem Sturz Fujimoris wieder voll in Kraft gesetzt worden. ${ }^{18}$ Mit 45765 Unterschriften gelang es der Initiative zur Wiederherstellung der Verfassung von 1979, das erforderliche Quorum von 0,3\% der Wahlberechtigten zu übertreffen. Der von ihr vorgelegte Gesetzentwurf erklärte die nach dem Staatsstreich erlassene Verfassung von 1993 für nichtig, sah aber auch Übergangsregeln vor, um ihrer faktischen Geltung Rechnung zu tragen. Zugleich sollten die Bestimmungen des Gesetzes 27600 zur Ausarbeitung einer neuen Verfassung aufgehoben werden; eine

Dies geschah durch die verfassungsändernden Gesetze 27680 von 2002 und 28607 von 2005. Auf dieser Grundlage wurden den in Anlehnung an die bestehenden Departements geschaffenen Regionen originäre Selbstverwaltungsbefugnisse übertragen; die geplante Zusammenfassung zu größeren Regionen scheiterte jedoch in einer Volksabstimmung am 30.10.2005.

Zahlreiche Beispiele für eine solche Einschränkung der Verfassung durch spätere Gesetze gibt Planas (Fn. 5), 573 f.; siehe auch unten bei Fn. 16, 18, 41, 50 f., 64, 69, 77.

Siehe zur Neuordnung des Justizsektors die Gesetze 27362, 27367 und 27368 von Oktober/ November 2000.

18

Zum Gesetz 26300 und seinen Einschränkungen unter Fujimori siehe Bennett-Ingold (Fn. 7), 124 ff., 128 ff.; diese wurden durch Gesetz 27520 von 2001 beseitigt, weitere Änderungen enthält das Gesetz 28421 von 2004. Vgl. jetzt zum Gesetz 26300 auch José Paulo Césare Sifuentes, Derechos de participación y control ciudadanos, NormLeg 365 (2006) II $181 \mathrm{ff}$. 
Reform der Verfassung von 1979 sei nur nach deren eigenen Regeln, ihre Ersetzung nur durch eine verfassunggebende Versammlung möglich. Dieser Gesetzentwurf wurde Anfang 2003 dem Kongress zugeleitet, der nach dem gesetzlich vorgesehenen Verfahren darüber in 120 Tagen zu befinden hatte. Stattdessen wurde der Entwurf an den Verfassungsausschuss überwiesen und dort nach drei Jahren archiviert. ${ }^{19}$

Das Gesetz 27600 mit seinen Bestimmungen zur Ausarbeitung einer neuen Verfassung war auch Gegenstand einer Klage der Anwaltskammer Cusco vor dem Verfassungsgericht, die freilich die Geltung der Verfassung von 1993 nicht in Frage stellte. Vielmehr wurde dieses Gesetz gerade deshalb als verfassungswidrig angegriffen, weil die darin ausgesprochene „Tilgung“ der Unterschrift Fujimoris eine unzulässige Rücknahme der Verkündung (despromulgación) der Verfassung von 1993 darstelle. Eine Reform dieser Verfassung könne nur nach deren Regeln, ihre Ersetzung nur durch eine verfassunggebende Versammlung erfolgen (das gleiche Argument also hier im umgekehrten Kontext). Mit der Anmaßung der Kompetenz zur Ausarbeitung einer neuen Verfassung habe der Kongress daher einen Staatsstreich begangen. In einem ausführlich begründeten Urteil wies das Verfassungsgericht diese Argumentation zurück. ${ }^{20}$ Für das Inkrafttreten der Verfassung von 1993 sei die Unterzeichnung durch den ,,japanischen Staatsbürger“ Fujimori nicht konstitutiv gewesen, da dieser nicht in seiner vorgeblichen Eigenschaft als verfassungsmäßiger Präsident, sondern als de-facto-Machthaber gehandelt habe. Durch die „Tilgung“ seiner Unterschrift könne daher die allein auf dem Willen des Verfassungsgesetzgebers beruhende Geltung der Verfassung nicht rückgängig gemacht werden. ${ }^{21}$ Diese sei zwar unter zweifelhaften Umständen zustande gekommen, bilde aber für das vorliegende Verfahren den rechtlichen Maßstab. Mit dem bloßen Auftrag an einen seiner Ausschüsse zur Ausarbeitung eines Verfassungsentwurfs habe der Kongress jedenfalls seine Kompetenzen nicht überschritten, da es dafür nicht einmal eines Gesetzes bedürfe. Eingehend erörtert das Gericht dann aber die Zulässigkeit und Gestaltung der geplanten Verfassungsreform, mit einem umfassenden Rückblick auf die peruanische Verfassungsgeschichte und unter Rückgriff insbesondere auf die deutsche Verfassungsrechtslehre. Entscheidend ist nach Auffassung des Gerichts, dass die Verfassung von 1993 neben den Regeln zur Verfassungsänderung selbst in Art. 32 ihre Totalreform als möglichen Gegenstand eines Referendums vorsehe. Wenn der Kongress eine solche Reform durch Verabschiedung eines Entwurfs vorbe2003-JNE dem Kongress zugeleitet, dort am 13.1.2003 an den Verfassungsausschuss überwiesen und von diesem am 30.3.2006 verworfen und archiviert (Of. $\mathrm{N}^{\circ}$ 027-2006/CCYR-CR).

20 Urteil v. 21.1.2003 - Exp. N ${ }^{\circ}$ 014-2002-AI/TC, abgedruckt in NormLeg $320 \S 281$. Das Urteil ist fälschlich vom 21.1.2002 datiert, wird aber in der Datenbank des Gerichts in der Rechtsprechung des Jahres 2003 geführt. 
reite, setze er sich damit nicht an die Stelle des Verfassungsgesetzgebers, der in diesem Fall unmittelbar durch das im vorgesehenen Referendum handelnde Volk verkörpert werde. ${ }^{22}$

Wurde die Geltung der Verfassung von 1993 in dieser Entscheidung noch ohne weiteres vorausgesetzt, so musste das Gericht schon bald ausführlich dazu Stellung nehmen. Diesmal handelte es sich um eine Klage von mehr als 5000 Bürgern mit dem Antrag, das so bezeichnete ,documento promulgado el 29 de diciembre de 1993 con el título de Constitución Política del Perú de 1993“ für verfassungswidrig zu erklären. ${ }^{23}$ Als Produkt eines defacto-Regimes fehle diesem Dokument die notwendige Legitimität einer Verfassung. Es habe auch keine tatsächliche Geltung als Verfassung erlangt, da seine Bestimmungen von seinen eigenen Urhebern ständig verletzt worden seien, die weder die Gewaltenteilung noch die Grundrechte respektiert hätten. Dagegen habe die Verfassung von 1979 nach ausdrücklicher Bestimmung ihres Art. 307 durch den gewaltsamen Umsturz ihre Geltung nicht verloren, weshalb das verfassungswidrige Dokument von 1993 für nichtig zu erklären sei. Das Gericht weist die Klage als unstatthaft zurück, lässt aber seine Sympathie mit dem Anliegen der Kläger erkennen. ${ }^{24}$ So teilt es deren Auffassung, dass die Verfassung von 1993 nicht in einem legitimen Verfahren zustande gekommen sei; ihre Geltung werde aber dadurch nicht berührt. Das Gericht weist in diesem Zusammenhang darauf hin, dass von den 12 peruanischen Verfassungen mit Ausnahme der Verfassung von 1979 alle ohne demokratische Legitimation in Kraft gesetzt wurden. Wenn die Verfassung von 1993 auch in den ersten Jahren ihres Bestehens von den Staatsorganen nicht respektiert worden sei, so werde sie doch seit dem Sturz Fujimoris von allen staatlichen Institutionen befolgt. Als Grundnorm im Sinne Kelsens sei sie der Maßstab für alles nachrangige Recht, könne aber selbst nicht an einer höherrangigen Norm gemessen werden. Insbesondere könne nicht die Verfassung von 1979 als Maßstab herangezogen werden, da diese nicht mehr in Kraft sei. ${ }^{25}$ Das Gericht betont, dass seine eigene Existenz wie auch das vorliegende Verfahren auf der Verfassung von 1993 beruhe, so dass deren Nichtigerklärung beidem die Grundlage entziehen würde. ${ }^{26}$ Auch könnten nur konkrete Normen mit Gesetzeskraft im Normenkontrollver-

Anders das abweichende Votum des Richters Aguirre Roca, der die Einberufung einer verfassunggebenden Versammlung für zwingend notwendig hält. Siehe auch Eloy Espinosa-Saldaña Barrera, Análisis crítico de la sentencia del Tribunal Constitucional sobre la Ley de Reforma total de la Constitución, RevPerJurispr 5 (2003) Nr. 25 S. 3 ff.

Gemäß Art. 203 Nr. 5 der Verfassung von 1993 kann die ,acción de inconstitucionalidad“ u.a. von 5000 wahlberechtigten Bürgern erhoben werden. Nach Art 299 Nr. 6 der Verfassung von 1979 waren dafür noch 50000 Unterschriften erforderlich.

Urteil v. 10.12.2003 - Exp. N 014-2003-AI/TC, abgedruckt in NormLeg $331 \S 209$ und RevPerJurispr 6 (2004) Nr. 35 S. 151 mit Anm. Carlos Hakansson Nieto, ebd. Nr. 38 S. 99 ff.

Das schließt es nach Ansicht des Gerichts aber nicht aus, die Urheber des Staatsstreichs nach Art. 307 der Verfassung von 1979 zu bestrafen, da sie damals geltendes Recht gewesen sei. 
fahren nach Art. 200 Nr. 4 der Verfassung überprüft werden; dazu gehöre weder die Verfassung als Ganzes noch ein irgendwie geartetes „Dokument““ ${ }^{27}$

Das Gericht versteht sich jedoch nicht nur als juristischer Spruchkörper, sondern zugleich als politisches Organ. Als solches teilt es die Bedenken der Kläger gegen die Verfassung von 1993; dieses drängende Problem könne aber nur auf institutionellem Wege gelöst werden. Deshalb greift das Gericht zu einer ungewöhnlichen Maßnahme: Im gleichen Tenor der Entscheidung, in dem es die Klage verwirft, fordert es den Kongress auf, die Verfassungsfrage noch in der laufenden Legislaturperiode einer endgültigen Lösung zuzuführen. ${ }^{28}$ Dieser Appell blieb freilich ungehört - der Kongress war zu einer klaren Entscheidung nicht in der Lage. ${ }^{29}$ Erst im Wahlkampf 2006 wurde die Frage von den beiden stärksten Parteien, der UPP und der APRA, wieder aufgegriffen; doch wird sie in der Regierungserklärung des neuen Präsidenten Alan García Pérez (APRA) nicht erwähnt. Dagegen wurden aus dem Kreis der UPP im neuen Parlament zwei Gesetzentwürfe eingebracht, die auf die Nichtigerklärung der Verfassung von 1993 sowie auf Wiederherstellung und Reform der Verfassung von 1979 zielen. ${ }^{30}$ Sie wurden an den Verfassungsausschuss überwiesen und erwarten dort ihr Schicksal; angesichts der Stimmenverhältnisse erscheint eine baldige Verabschiedung wenig wahrscheinlich. ${ }^{31}$

\section{Neuregelung der Verfassungsgerichtsbarkeit}

Das durch die Verfassung von 1979 geschaffene Verfassungsgericht (Tribunal de Garantías Constitucionales) hatte in der ersten Amtsperiode Fujimoris mehrere Gesetze seines Reformprogramms für verfassungswidrig erklärt. Dieser hatte daher unmittelbar nach seinem „Selbstputsch“ die Reorganisation des Gerichts verfügt und die amtierenden Richter

ren, auf dem seine Einrichtung beruhte: C.S.J. 7.1.1992, voto $\mathrm{N}^{\circ}$ 1-92, Boletín Judicial v. 1.12.1992, S. 3.

27 Der Richter Aguirre Roca hielt in seinem abweichenden Votum das gesamte Verfahren vor dem Verfassungsgericht für nichtig, da dasselbe zum Schutz der Verfassung berufen und damit für eine Klage wie die vorliegende absolut unzuständig sei.

In der Presseerklärung des Gerichts vom 18.12.2003, wiedergegeben in RevPerJurispr 6 (2004) Nr. 35 S. 151 f., wird diesem Teil der Entscheidung breiter Raum eingeräumt, die Klagabweisung dagegen nur kurz erwähnt. Siehe bei Fn. 13.

Gesetzentwürfe 73-2006-CR v. 28.8.2006 und129-2006-CR v. 6.9.2006. Die Reform der Verfassung von 1979 soll nach dem ersten Entwurf durch eine verfassunggebende Versammlung, nach dem zweiten durch den Kongress in Verbindung mit einem Referendum erfolgen. 
entlassen. ${ }^{32}$ Das heutige Verfassungsgericht (Tribunal Constitucional) wurde dann entgegen der ursprünglichen Absicht der Regierung in die Verfassung von 1993 aufgenommen und im Gesetz 26435 von 1995 näher geregelt; eine Überleitung der beim früheren Verfassungsgericht anhängigen Klagen war darin nur teilweise vorgesehen. ${ }^{33}$ Tatsächlich wurde das Gericht erst Mitte 1996 mit Richtern besetzt, war aber in seiner praktischen Wirksamkeit durch gesetzliche und faktische Eingriffe weitgehend beschränkt. Es kam schließlich zum Konflikt, als drei Verfassungsrichter sich in einer umstrittenen Entscheidung offen gegen die zweite Wiederwahl Fujimoris stellten und deshalb im Mai 1997 vom Kongress abgesetzt wurden. ${ }^{34}$ Nach dem politischen Umschwung wurden die Richter im November 2000 wieder in ihr Amt eingesetzt und so die Handlungsfähigkeit des Gerichts wiederhergestellt. ${ }^{35}$ Gleichwohl wurde allgemein ein dringendes Bedürfnis nach einer umfassenden Neuregelung dieser Materie empfunden. Diese erfolgte einerseits durch ein neues Organisationsgesetz für das Verfassungsgericht (Ley Orgánica del Tribunal Constitucional LOTConst), anderseits durch ein eigenes Verfassungsprozessgesetzbuch (Código Procesal Constitucional - CPConst), die beide Ende 2004 in Kraft getreten sind. ${ }^{36}$ Dabei handelt es sich allerdings nicht um einen völligen Neuanfang, sondern um eine geschlossene Kodifikation auf der Grundlage des bisherigen Rechts mit dem Ziel einer funktionsfähigen Verfassungsgerichtsbarkeit.

\section{Verfassungsgericht}

Das Verfassungsgericht findet seine rechtliche Grundlage in Art. 201 der Verfassung von 1993. Es besteht aus sieben Mitgliedern, die vom Kongress mit Zweidrittelmehrheit für

Dekret-Gesetze 25418 und 25422 vom April 1992. Näher dazu César Landa, Constitutional Justice in Peru, JöR 44 (1996) 583, 587 ff.; Jürgen Saligmann, Verfassungsgerichtsbarkeit in Lateinamerika, in: Helen Ahrens / Detlef Nolte (Hrsg.), Rechtsreformen und Demokratieentwicklung in Lateinamerika, 1999, 133, 137 f.; Norbert Lösing, Die Verfassungsgerichtsbarkeit in Lateinamerika, 2001, 355.

Siehe die Übergangsbestimmungen 5 und 7. Die Verfahren der abstrakten Normenkontrolle wurden archiviert; für die übrigen Verfahren setzte das Gesetz 26853 von 1997 eine Ausschlussfrist von 60 Werktagen.

Eingehend dazu Francisco Fernández Segado, El control normativo de la constitucionalidad en el Perú: Crónica de un fracaso anunciado, AnDerConstLatinoam 1999, 353, 382 ff. = BolMexDerComp 32 (1999) 765, 803 ff.; McFarland Sánchez-Moreno (Fn. 5), 584 ff.; vgl. auch Saligmann (Fn. 32), 141, 144, und unten Fn. 53. Die Absetzungen wurden erfolglos im Amparo-Verfahren angegriffen, Trib.Const. 10. und 16.7.1998, NormLeg 268, A-1 und A-11; zur Klage vor dem Interamerikanischen Gerichtshof siehe unten Fn. 123.

Resolución Legislativa 007-2000 v. 17.11.2000, NormLeg 294 § 126.

36

Ley Orgánica del Tribunal Constitucional: Gesetz 28301 v. 22.7.2004 (geändert durch Gesetz 28764 und 28943 von 2006). Código Procesal Constitucional: Gesetz 28237 v. 28.5 .2004 (geändert durch Gesetz 28642 von 2005 und Gesetz 28946 von 2006). 
fünf Jahre gewählt werden; eine unmittelbare Wiederwahl ist ausgeschlossen. ${ }^{37}$ Sie müssen die gleichen Voraussetzungen erfüllen wie die Richter des Obersten Gerichtshofs, also Peruaner von Geburt und über 45 Jahre alt sowie mindestens 10 Jahre als Richter oder Staatsanwalt an einem Obergericht oder 15 Jahre als Rechtsanwalt oder Rechtsprofessor tätig gewesen sein. Richter und Staatsanwälte können aber nur zu Verfassungsrichtern gewählt werden, wenn sie ihr Amt ein Jahr zuvor niedergelegt haben. Entfallen ist die Bestimmung der Verfassung von 1979, wonach als Verfassungsrichter nur Personen in Betracht kamen, die sich durch ihre demokratische Haltung und ihren Einsatz für die Menschenrechte ausgezeichnet hatten. ${ }^{38}$ Nach Art. 202 der Verfassung ist das Gericht zuständig für die abstrakte Normenkontrolle von Gesetzen, für Organstreitigkeiten sowie als letzte Instanz gegen ablehnende Entscheidungen bei Habeas-corpus-Verfahren und anderen in der Verfassung vorgesehenen Rechtsschutzklagen.

Das Organisationsgesetz für das Verfassungsgericht, das Gesetz 28301 von 2004, regelt Aufbau und Funktionen des Gerichts sowie die Rechtsverhältnisse der Richter und ihrer Hilfskräfte, enthält aber anders als seine Vorgänger keine Vorschriften für das Verfahren. ${ }^{39}$ Der (symbolische) Sitz des Verfassungsgerichts befindet sich in Arequipa, doch können Sitzungen durch Mehrheitsbeschluss auch an jedem anderen Ort in Peru - in der Praxis vorwiegend in Lima - anberaumt werden (Art. 1). Das Gericht kann seine Arbeitsweise durch Reglemente festlegen (Art. 2). ${ }^{40}$ Die wichtigsten Änderungen gegenüber der früheren Rechtslage betreffen die Beschlussfähigkeit des Gerichts. Nach dem unter Fujimori erlassenen Gesetz 26435 von 1995 war dafür die Anwesenheit von sechs der sieben Richter erforderlich; um eine Norm für verfassungswidrig zu erklären, bedurfte es zudem einer Mehrheit von sechs Stimmen. Wurde diese Mehrheit nicht erreicht, so war die Klage auf Feststellung der Verfassungswidrigkeit als unbegründet zurückzuweisen. Auf diese Weise konnte die Nichtigerklärung einer Norm schon durch zwei regierungstreue Richter verhindert wer-

Zum Vergleich: Nach der Verfassung von 1979 bestand das Gericht aus neun Richtern, von denen jeweils drei vom Kongress, von der Regierung und vom Obersten Gericht bestimmt wurden (Art. 296); zur Problematik dieses Wahlmodus siehe Saligmann (Fn. 32), 139.

Das Wahlverfahren regelt Art. 8 LOTConst, geändert durch Gesetz 28764 von 2006; dazu Césare Sifuentes, Conformación de los magistrados del Tribunal Constitucional, NormLeg 362 (2006) II 77 ff. Die letzte Wahl der Verfassungsrichter im Juni 2007 war von Unregelmäßigkeiten überschattet, die zu ihrer teilweisen Annulierung durch den Kongress führten; siehe zur Begründung den entsprechenden Resolutionsentwurf 1388/2006-CR v. 14.6.2007.

Siehe Fn. 36. Dagegen enthielten sowohl das Gesetz 23385 von 1982 (Ley Orgánica del Tribunal de Garantías Constitucionales) wie das Gesetz 26435 von 1995 (Ley Orgánica del Tribunal Constitucional) auch Vorschriften über die Normenkontrollklage und weitere Verfahren; diese finden sich jetzt im Verfassungsprozessgesetzbuch (unten II-III).

40

Reglamento Normativo del Tribunal Constitucional v. 14.9.2004, NormLeg $341 \S 030$ (zu späteren Änderungen ebd. $347 \S 279$ und $356 \S 264$ sowie unten Fn. 129); Reglamento de Organización y Funciones del Tribunal Constitucional, Resolución Administrativa 047-2006-P/TC v. 24.3.2006. 
den ${ }^{41}$ und war seit Mai 1997 wegen der Absetzung dreier Verfassungsrichter überhaupt ausgeschlossen. ${ }^{42}$ Die Regelung wurde vergeblich im Wege der Verfassungsklage angegriffen $^{43}$ und erst nach dem Sturz Fujimoris durch die Gesetze 27780 und 27850 von 2002 geändert. ${ }^{44}$ Nach dem geltenden Organisationsgesetz genügen nunmehr sowohl für die Beschlussfähigkeit wie für die Feststellung der Verfassungswidrigkeit fünf Richter; eine Stimmenthaltung ist entgegen früherer Praxis nicht zulässig (Art. 5). ${ }^{45}$

\section{Verfassungsprozess}

Durch Gesetz 28237 von 2004 wurde ein einheitliches Verfassungsprozessgesetzbuch, der „Código Procesal Constitucional“ geschaffen. ${ }^{46}$ Dieser regelt nicht nur die Verfahren vor dem Verfassungsgericht, sondern alle auf der Verfassung beruhenden Verfahren, auch soweit andere Gerichte dafür zuständig sind. In einem einleitenden Titel werden die Ziele und Grundprinzipien des Verfassungsprozesses festgelegt. ${ }^{47}$ Wesentliche Ziele des Verfassungsprozesses sind danach die Durchsetzung des Vorrangs der Verfassung und die tatsächliche Geltung der verfassungsmäßigen Rechte (Art. II). Zu den Grundprinzipien gehören die Offizialmaxime, der kostenfreie Zugang (was die Verurteilung in die Kosten nicht

So im Fall der von Fujimori betriebenen Justizreform; siehe Trib.Const. 29.10.1996, NormLeg 246, J-5 zu den entsprechenden Bestimmungen des Gesetzes 26623 von 1996.

42

Siehe oben bei Fn. 34. Das Gesetz 26801 von 1997 ermöglichte zwar die Beschlussfassung durch vier Richter, aber mit Ausnahme der Normenkontrollklage.

Die Klage wurde durch Entscheidung des Verfassungsgerichts vom 19.12.1996 zurïckgewiesen, Saligmann (Fn. 32), 143 f.; eingehend dazu Fernández Segado (Fn. 34), 373 ff. bzw. 791 ff.

Das Gesetz 27780 verlangte für die Beschlussfähigkeit 6 Richter, für die Feststellung der Verfassungswidrigkeit 5 Stimmen. Das Gesetz 27850 enthielt bereits eine dem jetzigen Art. 5 CPConst entsprechende Regelung (siehe den folgenden Text).

Eine Sonderregelung gilt für die Beschlussfassung bei Rechtsmittelentscheidungen in Amparound Habeas-corpus-Verfahren; siehe bei Fn. 61.

Siehe Fn. 36. Zu Entstehung und Inhalt näher Raúl Chanamé Orbe, El Código Procesal Constitucional, NormLeg 345 (2005) II 11 ff.; Luis R. Sáenz Dávalos, Los órganos competentes en el modelo de jurisdicción constitucional desarrollado por el Código Procesal Constitucional, RevPerJurispr 7 (2005) Nr. 48 S. 117 ff.; Francisco José Eguiguren Praeli, El nuevo Código Procesal Constitucional peruano, AnDerConstLatinoam 11 (2005) 331 ff.; Juan Bautista Bardelli Lartirigoyen, El nuevo Código Procesal Constitucional del Perú: una visión introspectiva, ebd. 353 ff.; Aníbal Quiroga León, El derecho procesal constitucional en el Perú y el Código Procesal Constitucional, ebd. 12 (2006) $377 \mathrm{ff}$.

47 Näher dazu Víctor Julio Ortecho Villena, El Título Preliminar del Código de las Libertades, NormLeg 340 (2004) II 15 ff.; José Paulo Césare Sifuentes, Principios constitucionales en el Título Preliminar del Código Procesal Constitucional, ebd. 351 (2005) II 135 ff.; Alexander Rioja Bermúdez, El Título Preliminar en el Nuevo Código Procesal Constitucional, RevJurPerú 55 (2005) Nr. 60 S. 167 ff. 
ausschließt) ${ }^{48}$ sowie der Grundsatz der Unmittelbarkeit; auch die Prozessformalitäten sollen entsprechend den Zielen des Verfassungsprozesses gehandhabt werden (Art. III). Bei der Rechtsanwendung ist der Richter nicht an den Vortrag der Parteien gebunden; für das Verfahren kann er hilfsweise auf andere Prozessgesetze, auf allgemeine Grundsätze des Prozessrechts sowie auf Rechtsprechung und Lehre zurückgreifen (Art. VIII-IX). Die Auslegung der von der Verfassung geschützten Rechte soll sich an der Allgemeinen Erklärung der Menschenrechte, den Verträgen über Menschenrechte sowie an den Entscheidungen der internationalen Menschenrechtsgerichte orientieren, die auf von Peru angenommenen Verträgen beruhen (Art. V).

Eine besondere Vorschrift betrifft die sog. „diffuse Kontrolle“ der Verfassungsmäßigkeit, die nicht nur dem Verfassungsgericht, sondern jedem Richter obliegt (im Gegensatz zu der beim Verfassungsgericht konzentrierten Kontrolle). Diese Möglichkeit ist in der Verfassung von 1993 ebenso wie in der früheren Verfassung von 1979 ausdrücklich vorgesehen und auch gesetzlich seit langem anerkannt. ${ }^{49}$ Danach darf der Richter keine Norm anwenden, die er für verfassungswidrig hält. Nach dem „Selbstputsch“ Fujimoris wurde diese konkrete Normenkontrolle zunächst für die von der Verwaltung erlassenen Vorschriften suspendiert $^{50}$ und später auch für bestimmte gesetzliche Normen einfach verboten. ${ }^{51}$ Dagegen scheiterte ein Gesetzentwurf, mit dem die konkrete Normenkontrolle generell in eine (aussichtslose) Normenkontrolle vor dem Verfassungsgericht umgewandelt werden sollte. ${ }^{52}$ Die umgekehrte Konstellation ergab sich, als im Jahre 1997 das Verfassungsgericht im Wege der abstrakten Normenkontrolle mit dem Gesetz 26657 befasst wurde, das die zweite Wiederwahl Fujimoris ermöglichen sollte. Da die zur Nichtigerklärung des Gesetzes benötigte qualifizierte Mehrheit von sechs Stimmen nicht erreicht werden konnte, versuchten einige Verfassungsrichter, in diesem Verfahren die „Nichtanwendbarkeit“ des Gesetzes im Wege der „diffusen“ Normenkontrolle festzustellen, was zu ihrer Absetzung durch den Kongress und der Aufhebung ihrer Entscheidung im Amparo-Verfahren durch den Obers-

Art. 138 Abs. 2 Verf. 1993; Art. 236 Verf. 1979. Ebenso bereits in Art. XXII des Zivilgesetzbuchs von 1936; später in den Gerichtsverfassungsgesetzen von 1963 (Art. 8) und 1991 (Art. 14). Entsprechende Vorschriften finden sich in den Schlussvorschriften der Organisationsgesetze für das Verfassungsgericht von 1995 und 2004.

Das Gesetz 24968 von 1988 über die Popularklage enthielt in seinem Art. 7 einen ausdrücklichen Hinweis auf die „diffuse Kontrolle“, der durch Gesetz 25433 von 1992 aufgehoben wurde.

Siehe die Amnestiegesetze 26479 und 26492 von 1995 und dazu den spektakulären Fall bei Lösing (Fn. 32), 357 f. rigkeit einer Norm sie dem Obersten Staatsanwalt (Fiscal de la Nación) zuleiten und dieser dann die ,acción de inconstitucionalidad“ vor dem Verfassungsgericht erheben. 
ten Gerichtshof führte. ${ }^{53}$ Dieses Vorgehen der Richter ist freilich mit dem Sinn der konkreten Normenkontrolle nicht vereinbar und nur durch die damaligen politischen Umstände zu erklären. $^{54}$

Nunmehr hat das Verfassungsprozessgesetzbuch in seinem Art. VI die Voraussetzungen der „diffusen“ Normenkontrolle klarer formuliert: Im Konflikt zwischen der Verfassung und einer niederrangigen Norm muss der Richter der ersteren den Vorzug geben, ,sofern es für die Entscheidung des Rechtsstreits darauf ankommt“. Auch muss er zunächst versuchen, den Konflikt im Wege der verfassungskonformen Auslegung zu lösen. ${ }^{55}$ Bei seiner Entscheidung muss der Richter auch die Rechtsprechung des Verfassungsgerichts berücksichtigen und darf insbesondere die Anwendung einer Norm nicht ablehnen, deren Verfassungswidrigkeit in einem Normenkontrollverfahren verneint worden ist. Das Verfassungsgericht kann darüber hinaus seinen eigenen Entscheidungen bindende Präzedenzwirkung verleihen; von einer solchen Entscheidung kann das Gericht selbst nur abweichen, wenn es die dafür maßgebenden tatsächlichen und rechtlichen Gründe benennt (Art. VII). Für die konkrete Normenkontrolle ist weiterhin die Vorschrift des Art. 14 des Gerichtsverfassungsgesetzes von 1991 zu beachten: Danach muss der Richter, der eine Norm mit Gesetzesrang für verfassungswidrig hält, seine nicht mit Rechtsmitteln angefochtene oder anfechtbare Entscheidung dem Obersten Gerichtshof zur Überprüfung vorlegen. ${ }^{56}$

\section{Verfassungsklagen}

Neben der inzidenten Verfassungskontrolle enthält das Verfassungsprozessgesetzbuch eine ausführliche Regelung derjenigen Verfahren, für welche die Verfassung von 1993 selbst in

Siehe die beiden Entscheidungen des Verfassungsgerichts vom 3.1.1997 in RevJurPerú 46 (1996) Nr. 4 S. 40 ff., 49 ff., und die des Obersten Gerichtshofs vom 30.12.1997, ebd. 48 (1998) Nr. 15 S. 281 ff. mit kritischer Anmerkung Milagros Maraví Sumar, ebd. 285 ff.; dazu die Autoren oben Fn. 34.

Kritisch auch Fernández Segado (Fn. 34), 379 f. bzw. 799; Alfredo Quispe Correa, Tribunal Constitucional ¿Solución o confusión?, RevJurPerú 46 (1996) Nr. 4 S. 27 ff.; Sigifredo Orbegoso $V .$, El tortuoso camino "legal" de una reelección más, ebd. S. 38-1, 38-10 ff.; zustimmend dagegen aus politischen Gründen Aníbal Quiroga León, A propósito del "control difuso" o "judicial review" en Perú, ebd. 33 ff.

So bereits der Oberste Gerichtshof in einer Entscheidung vom 12.8.1991, NormLeg 222, J-7; ebenso die oben genannten Bestimmungen der Organisationsgesetze für das Verfassungsgericht von 1995 und 2004 (Fn. 49).

56

Diese Regelung stammt aus dem Gerichtsverfassungsgesetz von 1963 (Fn. 49), als es noch kein Verfassungsgericht gab; zum Nebeneinander dieser Vorschrift und Art. VI CPConst siehe Quiroga León (Fn. 46), 391 f. In das Verfassungsprozessgesetzbuch wurde eine entsprechende Regelung erst durch Gesetz 28946 von 2006 (an der falschen Stelle) bei den Rechtsschutzklagen als Art. 3 Abs. 3-4 eingefügt, die aber ebenso in anderen Prozessen gilt. 
Art. 200 eine eigene Klageform vorsieht. Dazu gehören einerseits diejenigen Klagen, die zur Durchsetzung verfassungsmäßiger oder gesetzlicher Rechtspositionen dienen, anderseits die Klagen zur abstrakten Normenkontrolle gegen gesetzliche oder nachrangige Vorschriften. Daneben werden im Verfassungsprozessgesetzbuch auch die sog. Kompetenzkonflikte sowie der Zugang zur internationalen Gerichtsbarkeit geregelt. Schließlich finden sich in den abschließenden Titeln noch einige Vorschriften über das Verfahren vor dem Verfassungsgericht sowie bei sonstigen Verfassungsklagen; hervorzuheben ist die Bestimmung, wonach die Entscheidungen des Verfassungsgerichts vor keinem anderen nationalen Gericht angefochten werden können (Art. 121). ${ }^{57}$

\section{Rechtsschutzklagen}

Die Rechtsschutzklagen der Verfassung umfassen die traditionellen Habeas-corpus- und Amparo-Verfahren ebenso wie die in neuerer Zeit hinzugekommenen Verfahren des Habeas data und die Erfüllungsklage. Für alle diese Verfahren enthält das Verfassungsprozessgesetzbuch in seinem ersten Titel gemeinsame Vorschriften. ${ }^{58}$ Dieser Titel (Art. 1-24) beruht inhaltlich auf dem Gesetz 23506 von 1982, in dem ursprünglich das Habeas-corpusund Amparo-Verfahren geregelt war, enthält jedoch wesentliche Änderungen gegenüber dem früheren Rechtszustand. Die folgenden Titel sind den einzelnen Verfahren gewidmet; auch die ,gemeinsamen“ Vorschriften gelten aber teilweise nur für bestimmte Verfahren. Mit Ausnahme des Habeas-corpus-Verfahrens sind die Rechtsschutzklagen nunmehr subsidiär gegenüber anderen Rechtsbehelfen. ${ }^{59}$ Zuständig für die Rechtsschutzklagen sind wie bisher die ordentlichen Gerichte; der Rechtszug geht jetzt nicht mehr bis zum Obersten Gerichtshof, vielmehr ist bereits gegen die ablehnende Entscheidung der zweiten Instanz ein Rechtsmittel zum Verfassungsgericht gegeben. ${ }^{60}$ Dieses entscheidet dann durch einen

Vgl. dazu José Paulo Césare Sifuentes, Disposiciones generales de los procesos de hábeas corpus, amparo, hábeas data y cumplimiento, NormLeg 352 (2005) II $117 \mathrm{ff}$.

Vgl. Art. 5 Nr. 2-4 CPConst. Damit sollte vor allem die hohe Zahl der Amparo-Verfahren im Arbeitsrecht eingedämmt werden; näher dazu Christian Donayre Montesinos, Implicancias del Código Procesal Constitucional peruano: la consagración de un amparo residual y el nuevo escenario para la tutela de los derechos constitucionales laborales, Derecho 58 (2005) 333 ff.; Carolina Castillo Pastor, La procedencia de la acción de amparo a la luz de la incorporación del inciso 2 del artículo 5 del Código Procesal Constitucional, NormLeg 353 (2005) II 163, 167 ff.; Carlos Alberto Quispe Montesinos, La residualidad del proceso de amparo frente a la tutela de los derechos constitucionales laborales, RevPerJurispr 8 (2006) Nr. 66 S. 21 ff.

60

Die Verfassungsbeschwerde (recurso de agravio constitucional) muss vom Präsidenten des zweitinstanzlichen Gerichts zugelassen werden (Art. $18 \mathrm{CPConst).} \mathrm{Andernfalls} \mathrm{ist} \mathrm{innerhalb} \mathrm{von} \mathrm{fünf}$ Tagen die Nichtzulassungsbeschwerde (recurso de queja) zum Verfassungsgericht möglich (Art. 19 CPConst). In einem Ausnahmefall kann auf diesem Wege auch eine stattgebende Entscheidung angefochten werden, vgl. Trib.Const. 19.4.2007, RevJurPerú 2007 Nr. 76 S. 40 (42). 
der beiden dafür eingerichteten und mit drei Richtern besetzten Senate, deren Beschluss einstimmig ergehen muss, innerhalb einer Frist von 20 bzw. 30 Tagen. ${ }^{61}$ Dabei handelt es sich um eine Entscheidung in der Sache, nur bei prozessualen Verstößen ist eine Rückverweisung an das Instanzgericht vorgesehen (Art. 20). ${ }^{62}$ Eine vorläufige Vollstreckung während des Rechtsmittelverfahrens ist in bestimmten Fällen möglich. ${ }^{63}$ Dem vor den ordentlichen Gerichten unterlegenen Staat steht nach der Verfassung kein weiteres Rechtsmittel zu, was unter der Regierung Fujimori zeitweise durch Dekret-Gesetz geändert worden war. ${ }^{64}$ Im Gegensatz zum früheren Recht wird auch während des Ausnahmezustands der verfassungsrechtliche Rechtsschutz nicht ausgeschlossen, sondern in einer differenzierten Regelung nach dem Grundsatz der Verhältnismäßigkeit beschränkt. ${ }^{65}$

\section{a) Habeas corpus}

Der klassische Rechtsbehelf zum Schutz der persönlichen Freiheit ist das Habeas-corpusVerfahren, welches in Peru bereits seit Ende des 19. Jahrhunderts existiert. ${ }^{66}$ Seine praktische Bedeutung war allerdings stets hinter den damit verbundenen Erwartungen zurückgeblieben. ${ }^{67}$ Unter der Verfassung von 1979 bildete das Gesetz 23506 von 1982 die maßgebende Rechtsgrundlage. Dieses Gesetz wurde unmittelbar nach dem Amtsantritt der Regierung Fujimori durch ein restriktives Reglement ,ergänzt“, dessen ausdrückliches Ziel

Diese Regelung findet sich schon in dem Verfassungsgerichtsgesetz von 1995 (Gesetz 26435, Art. 42); das Verfassungsgerichtsgesetz von 1982 sah demgegenüber eine reine Kassationsentscheidung vor (Gesetz 23385, Art. 46);

Näher dazu Juan José Santivañez Antúnez, El cumplimiento anticipado de sentencia impugnada en el Código Procesal Constitucional, RevPerJurispr 7 (2005) Nr. 57 S. 35 ff.; Alexander Rioja Bermudez, Actuación de la sentencia impugnada en el proceso constitucional de amparo, NormLeg 360 (2006) II 147 ff.; einschränkend Fabrizio Castellano Brunello, La irreversibilidad: Límite a la actuación del proceso de amparo conforme al Código Procesal Constitucional, NormLeg 346 (2005) II 263 ff.

Siehe Art. 298 Nr. 2 Verf. 1979; Art. 202 Nr. 2 Verf. 1993. Das berüchtigte Dekret-Gesetz 25721 von 1992 zielte demgegenüber darauf ab, dem Staat im Amparo-Verfahren die Anrufung des faktisch nicht existenten Verfassungsgerichts und damit die Suspendierung des Verfahrens zu ermöglichen (aufgehoben durch Gesetz 26435 von 1995).

Art. 200 Abs. 3-4 Verf. 1993; Art. 23 CPConst. Anders das frühere Gesetz 23506 von 1982 über das Habeas-corpus- und Amparo-Verfahren in seinem Art. 38; siehe dazu die Entscheidung des Obersten Gerichtshofs vom 23.7.1986, RevJurPerú 39 (1988) 491. Belaunde, El Hábeas Corpus interpretado (1933-1970), 1971, 11 ff.

Siehe zur älteren Praxis García Belaunde (Fn. 66), 14 ff; für die Zeit ab 1979 insbesondere Comisión Andina de Juristas, Perú y Chile, Poder judicial y derechos humanos, 1988, 51 ff., und die Übersicht bei Landa (Fn. 32), 592; ferner Franciso José Eguiguren Praeli, La libertad individual y su protección judicial mediante la acción de habeas corpus, in: Comisión Andina de Juristas, Lecturas sobre temas constitucionales 2, 1988, 13 ff., und die Nachweise bei Lösing (Fn. 32), 354. 
in der Eindämmung der verfassungsrechtlichen Rechtsschutzklagen bestand. ${ }^{68}$ Demgegenüber bestand der Kongress auf einer gesetzlichen Regelung, die er auch gegen das Veto des Präsidenten verabschiedete (Gesetz 25398 vom Februar 1992). Nach dessen „Selbstputsch“ wurde das Habeas-corpus-Verfahren im Rahmen der Terrorismusbekämpfung zeitweise völlig ausgeschlossen, später mit Beschränkungen wieder zugelassen und auch unter der Verfassung von 1993 durch gesetzgeberische Maßnahmen weiter eingeschränkt. ${ }^{69}$ Diese gesetzlichen Regelungen wurden nach dem Sturz Fujimoris z.T. vom Verfassungsgericht für unwirksam erklärt ${ }^{70}$ und schließlich durch das Verfassungsprozessgesetzbuch von 2004 insgesamt aufgehoben.

Nach Art. 200 Nr. 1 der Verfassung von 1993 ist die Habeas-corpus-Klage zulässig gegen Handlungen oder Unterlassungen einer Behörde, eines Amtsträgers ${ }^{71}$ oder einer sonstigen Person, welche die persönliche Freiheit oder die damit verbundenen verfassungsmäßigen Rechte verletzen oder bedrohen. Welche der in Art. 2 der Verfassung verbürgten Grundrechte dazu gehören, wird im Einzelnen durch Art. 25 des Verfassungsprozessgesetzbuchs festgelegt. Der Katalog der dort aufgeführten Rechte unterscheidet sich nur in wenigen Punkten von der früheren Regelung im Gesetz über das Habeas-corpus- und Amparo-Verfahren. Ausdrücklich umfasst von der Habeas-corpus-Klage ist nunmehr auch das Folterverbot, das Recht auf angemessene Behandlung als Gefangener, der Schutz vor zwangsweiser Einziehung zum Militärdienst sowie vor „gewaltsamem Verschwinden“ (desaparición forzada). $^{72}$ Die Habeas-corpus-Klage kann von dem Betroffenen oder einem Dritten erhoben werden, der dazu keiner Vollmacht bedarf (Art. 26). Sie ist nicht an eine bestimmte Form gebunden und kann auch auf elektronischem oder sonstigem Wege eingelegt werden (Art. 27). ${ }^{73}$ Zuständig ist in erster Instanz jeder Strafrichter unabhängig von der

D.S. 024-90-JUS v. 12.7.1990, NormLeg 173, S. 52: ,a fin de coadyuvar a una pronta y eficaz administración de justicia y ante el significativo volumen de acciones de garantía que muchas veces innecesaria e indebidamente se sustancian ante el Poder Judicial ..."

Siehe die Dekret-Gesetze 25659 und 26248 von 1992/93, später die Gesetzgebungsdekrete 824, 895 und 900 von 1996/98 sowie Gesetz 27235 von 1999.

Trib.Const.13.8.2001, NormLeg 309, A-7 zu Gesetzgebungsdekret 900 von 1998.

Zum Habeas corpus gegen Gerichtsentscheidungen siehe unten bei Fn. 90 f., 93 f.

Siehe dazu Fernando Vicente Núñez, Pérez, El hábeas corpus instructivo: Hacia la ubicación de las víctimas y la individualización de los responsables del delito de desaparición forzada como manifestaciones del derecho a la verdad, NormLeg 346 (2005) II 243 ff. Ein erster Bericht über die in der Regierungszeit Fujimoris ,verschwundenen“ Personen wurde bereits im November 2000 vom „Defensor del Pueblo“ veröffentlicht, Res. 57-2000/DP, NormLeg 294 § 127. Das Gesetz 28413 von 2004 regelt die zivilrechtlichen Verhältnisse dieser Personen; zu den strafrechtlichen Konsequenzen siehe Trib.Const. 9.12.2004, RevPerJurispr 7 (2005) Nr. 51 S. 284. 
Geschäftsverteilung (Art. 28) ${ }^{74}$; dieser kann im Fall räumlicher Entfernung auch den Friedensrichter am Aufenthaltsort des Festgenommenen zu entsprechenden Maßnahmen ermächtigen (Art. 29). Das wieder funktionsfähige Verfassungsgericht hat dazu inzwischen eine reichhaltige Kasuistik entwickelt; auf dieser Grundlage unterscheidet die Lehre heute 9 oder 10 verschiedene Formen des Habeas corpus. ${ }^{75}$ Eine eingehende Darstellung würde den Rahmen dieses Aufsatzes sprengen und muss deshalb späteren Untersuchungen überlassen bleiben.

b) Amparo

Das in vielen lateinamerikanischen Ländern verbreitete Rechtsinstitut des "amparo" wurde in Peru erstmals in der Verfassung von 1979 ausdrücklich anerkannt und sollte hier dem Schutz der verfassungsmäßigen Rechte dienen, die nicht vom Habeas-corpus-Verfahren umfasst waren (Art. 295). Seine systematische Regelung fand das Amparo-Verfahren dann in dem bereits genannten Gesetz 23506 von 1982 zusammen mit dem Habeas-corpus-Verfahren. ${ }^{76}$ Ebenso wie dieses wurde es nach dem Staatsstreich Fujimoris in vielfältiger Weise eingeschränkt. So wurde bei der Reorganisation und „Säuberung“ der Justiz den betroffenen Richtern und Staatsanwälten die Amparo-Klage durch Dekret-Gesetz abgeschnitten, was aber durch die Rechtsprechung des Verfassungsgerichts z.T. korrigiert wurde. $^{77}$ Auch in anderen Fällen wurde dieser Rechtsbehelf ausdrücklich ausgeschlossen, etwa bei der Aufhebung der Tankstellenkonzessionen, der Entlassung der Parlaments- oder Zollangestellten, den Zwangsmietverträgen zugunsten der Polizei sowie beim Abbau von bestehenden arbeits- oder sozialrechtlichen Ansprüchen. ${ }^{78}$ Ferner wurde durch DekretGesetz 25433 von 1992 der vorläufige Rechtsschutz im Amparo-Verfahren erschwert und von der Bestätigung durch die zweite Instanz abhängig gemacht.

Gegen diese Allzuständigkeit wendet sich wegen der daraus folgenden Missbräuche ein neuerer Gesetzentwurf der Regierung 1383/2006-PE v. 12.6.2007.

Siehe Christian Donayre Montesinos, Los tipos de hábeas corpus en la jurisprudencia del Tribunal Constitucional peruano y su tratamiento en el Código Procesal Constitucional, RevPerJurispr 7 (2005) Nr. 47 S. XIII ff.; Luis Castillo Córdova, La finalidad del Hábeas Corpus, ebd. Nr. 53 S. 31 ff.; Leny Palma Encalada, El proceso de Hábeas Corpus y su regulación en el nuevo diseño procesal constitucional, NormLeg 351 (2005) II 117 ff.; Luis Alberto Huerta Guerrero, El proceso constitucional de hábeas corpus en el Perú, RevJurPerú 55 (2005) Nr. 64 S. 185 ff. = AnDerConstLatinoam 12 (2006) 557 ff.

Zum Amparo-Verfahren unter der Verfassung von 1979 siehe Comisión Andina de Juristas (Fn. 67), $113 \mathrm{ff}$.

77

Vgl. Dekret-Gesetz 25454 von 1992 und weitere. Siehe dagegen Trib.Const. 13.6.1997, NormLeg 256, A-7; 12.1.2000, ebd. 287, A-27; nach dem Sturz Fujimoris noch 10.1.2001, ebd. 307, A-8; ebenso C.Sup.Lima 6.7.2001, ebd. 304, A-4.

Siehe die Dekret-Gesetze 25545, 25640, 25700, 25967, 25994, 26090 und 26119 von 1992. 
Auch nach Art. 200 Nr. 2 der Verfassung von 1993 bietet das Amparo-Verfahren komplementären Schutz zum Habeas-corpus-Verfahren, nämlich gegenüber solchen Handlungen oder Unterlassungen einer Behörde, eines Amtsträgers oder einer sonstigen Person ${ }^{79}$, welche die „übrigen“ verfassungsmäßigen Rechte verletzen oder bedrohen. Die Abgrenzung im Einzelnen ist dem Gesetzgeber überlassen; die konkrete Reichweite des Amparo-Verfahrens ergibt sich heute aus Art. 37 des Verfassungsprozessgesetzbuchs. ${ }^{80}$ Dabei kommt es gegenüber der früheren Regelung zu geringfügigen Verschiebungen. ${ }^{81}$ Dagegen können Rechte, die ihre Grundlage nicht in der Verfassung finden, im Amparo-Verfahren nicht geltend gemacht werden (Art. 38). Inwieweit auch Programmsätze der Verfassung eine Amparo-Klage rechtfertigen, ist nach den konkreten Umständen des Einzelfalles abzuwägen. ${ }^{82}$ Die Klage kann von dem Betroffenen oder seinem Bevollmächtigten erhoben werden (Art. 39-40); nur wenn dies aus tatsächlichen Gründen ausgeschlossen ist, kann auch ein Dritter die Klage erheben (Art. 41). Die Frist beträgt 60 Tage von dem Zeitpunkt, seit dem dem Betroffenen die Erhebung der Klage möglich war (Art. 44). Zuständig ist nach Wahl des Klägers der Zivil- oder Allgemeinrichter (juez civil o mixto) am Ort der Rechtsverletzung oder am Wohnsitz des Klägers; eine Prorogation ist ausgeschlossen (Art. 51). ${ }^{83}$ Eine offensichtlich unzulässige Klage kann vom Richter mit entsprechender Begründung sofort zurückgewiesen werden (Art. 47) ${ }^{84}$ - nicht aber vom Gerichtspersonal (Art. 42 Abs. 2)! Ist dagegen eine tatsächliche oder drohende Rechtsverletzung glaubhaft gemacht, so kann bei Gefahr im Verzug auch vorläufiger Rechtsschutz gewährt werden, der entgegen bisherigem

Zur Amparo-Klage gegen Privatpersonen siehe M.Abraham García Chávarri, Algunas observaciones acerca del amparo contra particulares, NormLeg 350 (2005) II 157, 172 ff.

Zum Amparo-Verfahren nach diesem Gesetzbuch siehe jetzt Eloy Espinosa-Saldaña Barrera, El Código Procesal Constitucional peruano y la procedencia o improcedencia del amparo, RevJurPerú 54 (2004) Nr. 59 S. 19 ff.; César Landa, El amparo en el nuevo Código Procesal Constitucional peruano, AnDerConstLatinoam 11 (2005) 361 ff.; José Paulo Césare Sifuentes, El proceso de amparo y su procedimiento, NormLeg 346 (2005) II 267 ff.; Klagmuster und Übersicht über den Verfahrensgang ebd. 348 (2005) II 213 ff., 352 (2005) II 151 f., 358 (2006) II 181 f. und 366 (2006) II 137 ff.; Beispiel einer Klagerwiderung in RevJurPerú 2007 Nr. 71 S. 20 f.

So war z.B. die Unverletzlichkeit der Wohnung nach dem Gesetz 23506 durch das AmparoVerfahren geschützt, während sie nunmehr nach Art. 25 CPConst dem Habeas-corpus-Verfahren unterfällt.

Zu dieser umstrittenen Frage siehe die ausführliche Studie von Luis R. Sáenz Dávalos, La protección procesal de los derechos constitucionales programáticos, RevPerJurispr 8 (2006) Nr. 62 S. 3 ff. mit Hinweisen auf die neuere Rechtsprechung des Verfassungsgerichts.

Die Neufassung des Art. 51 durch Gesetz 28946 von 2006 sollte die bisherigen Wahlmöglichkeiten beseitigen und Missbräuchen entgegenwirken. Für Klagen gegen Gerichtsbeschlüsse ist nach Abs. 6 die Corte Superior zuständig. 
Recht nicht der Bestätigung durch die zweite Instanz bedarf und bis zum Abschluss des Verfahrens fortbesteht (Art. 15). ${ }^{85}$

Unzulässig ist nach Art. 200 Nr. 2 der Verfassung von 1993 eine Amparo-Klage gegenüber Rechtsnormen oder Gerichtsentscheidungen, die aufgrund eines ordnungsgemäßen Verfahrens ergangen sind. Dadurch sollte einer Ausuferung des Amparo-Rechtsschutzes und seiner Entwicklung zum Super-Rechtsmittel begegnet werden. ${ }^{86}$ Gleichwohl hat die Rechtsprechung Amparo-Klagen gegen gesetzliche Regelungen zugelassen, wenn diese den Einzelnen unmittelbar und konkret in seinen verfassungsmäßigen Rechten berühren. ${ }^{87}$ Diese Klagen gegen sog. „,normas autoaplicativas“ sind in Art. 3 des Verfassungsprozessgesetzbuchs seit der Neufassung durch Gesetz 28946 von 2006 speziell geregelt. ${ }^{88}$ Das Verbot des amparo gegen Gerichtsentscheidungen nach einem ordnungsgemäßen Verfahren fand sich bereits früher im Gesetz 23506 von 1982 (Art. 6 Nr. 2); nach dem ergänzenden Gesetz 25398 von 1992 konnten auch Verfahrensfehler nur mit ordentlichen Rechtsmitteln gerügt werden (Art. 10). Die Rechtsprechung unterschied daher zwischen der Verletzung einfacher Verfahrensvorschriften (irregularidad procesal) und einem nicht ordnungsgemäßen Verfahren (procedimiento irregular). ${ }^{89}$ Nunmehr will Art. 4 des Verfassungsprozessgesetzbuchs Habeas-corpus- und Amparo-Klagen nur noch gegenüber solchen Entscheidungen zulassen, die offensichtlich die prozessualen Garantien eines effektiven

Art. 15 CPConst (idF. durch Gesetz 28946 von 2006) steht unter den „Gemeinsamen Vorschriften“, gilt aber nicht für das Habeas-Corpus-Verfahren. Vgl. dazu César Augusto Cotos López, El nuevo Código Procesal Constitucional y la protección de los derechos fundamentales mediante la adopción de medidas cautelares, RevPerJurispr 7 (2005) Nr. 50 S. XXXIII ff.

Nach einer Untersuchung der etwa 2000 veröffentlichten Amparo-Entscheidungen der Jahre 1982-86 handelte es sich in fast $60 \%$ der Fälle um Klagen gegen gerichtliche Entscheidungen; vgl. Samuel B. Abad Yupanqui, ¿Procede el amparo contra resoluciones judiciales?, in: Lecturas sobre temas constitucionales 2 (Fn. 67), 35ff. (37).

So ausdrücklich das Verfassungsgericht bei den durch formelles Gesetz verfügten Richterentlassungen (oben Fn. 77); ebenso schon früher C.S. 25.9.1992, NormLeg 243, J-8 zur Zwangseinziehung eines Grundstücks durch Gesetz; weitere Beispiele unten Fn. 109, 118. Siehe zu dieser Rechtsprechung auch Christian Guzmán Napurí, El amparo contra normas legales y el control difuso, NormLeg 295 (2000) A-65 ff.; Luis Castillo Córdova, Normas autoaplicativas, alternatividad y "amparo contra amparo" en el Código Procesal Constitucional, RevJurPerú 54 (2004) Nr. 59 S. 33 ff.; Ana Cristina Neyra Zegarra, ¿Procede el amparo contra leyes frente al silencio del Código Procesal Constitucional?, ebd. 56 (2006) Nr. 66 S. 193, 210 ff.

Sie zielen auf die Nichtanwendbarkeit der betreffenden Norm im konkreten Fall und lassen deren Geltung unberührt. gegen den Beschluss des Verfassungsgerichts stützte sich darauf, dass es sich dabei um ein irreguläres Verfahren gehandelt habe. Rechtsvergleichend zu diesen Fragen Andrés Arturo Churampo Garibaldi, Acción de amparo contra resoluciones judiciales en el Perú, RevPerJurispr 6 (2004) Nr. 39 S. 45 ff. mit weiteren Rechtsprechungshinweisen. 
Rechtsschutzes versagen. ${ }^{90}$ Das Verfassungsgericht hält jedoch eine solche Beschränkung auf die prozessualen Grundrechte nicht für zulässig: Auch bei einem richterlichen Verstoß gegen die materiellen Grundrechtsverbürgungen fehle es an einem ordnungsgemäßen Verfahren im Sinne der Verfassung, was deshalb mit der Amparo-Klage gerügt werden könne. ${ }^{91}$ Das entspricht der allgemeinen Ansicht des Gerichts, wonach kein Akt der öffentlichen Gewalt der verfassungsgerichtlichen Kontrolle entzogen werden kann. ${ }^{92}$

Generell ausgeschlossen sind dagegen Rechtschutzklagen gegen Entscheidungen, die in einem anderen Verfassungsprozess ergangen sind. Dieses Verbot des ,amparo contra amparo“ oder „hábeas corpus contra hábeas corpus“, ausdrücklich niedergelegt in Art. 5 Nr. 6 des Verfassungsprozessgesetzbuchs, war freilich in neuerer Zeit ebenfalls fragwürdig geworden. ${ }^{93}$ Das Verfassungsgericht hat nunmehr in einer Entscheidung, die Präzedenzwirkung nach Art. VII des Verfassungsprozessgesetzbuchs beansprucht, bindende Grundsätze aufgestellt, nach denen in diesen Fällen zu entscheiden ist. Eine Amparo-Klage gegen eine zweitinstanzliche Amparo-Entscheidung ist danach nur zulässig, wenn ein am Verfahren nicht beteiligter Dritten erstmals durch die Entscheidung beschwert wird oder wenn eine der Prozessparteien rechtlich oder tatsächlich gehindert war, ein Rechtsmittel zum Verfassungsgericht einzulegen. Voraussetzung dafür ist, dass die Entscheidung eine gravierende Verletzung nicht nur eines prozessualen, sondern auch eines materiellen Grundrechts enthält, was insbesondere bei einem Verstoß gegen die Rechtsprechung des Verfassungsgerichts der Fall ist. Gegen eine Entscheidung des Verfassungsgerichts kann eine AmparoKlage nicht erhoben werden. ${ }^{94}$

c) Habeas data

Das Habeas-data-Verfahren, in Südamerika nach spanisch-portugiesischem Vorbild erstmals in der brasilianischen Verfassung von 1988 geregelt, wurde auch in die peruanische

Vgl. entsprechende Klagen in RevJurPerú 2006 Nr. 70 S. 23 f. und 2007 Nr. 74 S. 20 f. Eine Änderung des Art. 4 CPConst für das Habeas-corpus-Verfahren fordert Javier A. Aguirre Ch., Hábeas corpus contra resoluciones judiciales penales en el Código Procesal Constitucional, Derecho 58 (2005) 293 ff., 307.

Entscheidung vom 18.2.2005 (Exp. 3179-2004-AA/TC). Eingehend dazu Mijail Mendoza Escalante, Tribunal Constitucional y control material de resoluciones judiciales, ebd. 2007 Nr. 73 S. 11 ff.; vgl. auch den kritischen Kommentar in RevJurPerú 2006 Nr. 70 S. 24 f.

Trib.Const. 8.11.2005, RevPerJurispr 7 (2005) Nr. 57 S. 219 und 21.7.2006, NormLeg 363 II 84 zur umstrittenen Frage der gerichtlichen Kontrolle der Entscheidungen der Wahlorgane. Einschränkend dagegen Gesetz 28642 v. 7.12.2005, für verfassungswidrig erklärt durch Trib.Const. 19.6.2007, NormLeg 379 § 232.

Für die Zulässigkeit einer solchen Klage bei Verletzung der prozessualen Garantien eines effektiven Rechtsschutzes gemäß Art. 4 CPConst siehe Castillo Córdova (Fn. 87), 43 ff. m.Nw., und den Fall in RevJurPerú 2007 Nr. 73 S. 26 f. 
Verfassung von 1993 übernommen. ${ }^{95}$ Nach Art. 200 Nr. 3 der Verfassung werden dadurch bestimmte Grundrechte geschützt, welche die informationelle Selbstbestimmung betreffen. Eine erste Regelung enthielt das Gesetz 26301 von 1994; später hat das Gesetz 26470 von 1995 die Abgrenzung gegenüber dem Amparo-Verfahren präzisiert. ${ }^{96}$ Heute richtet sich das Verfahren nach den Art. 61-65 des Verfassungsprozessgesetzbuchs ${ }^{97}$ und entspricht weitgehend dem Amparo-Prozess, kann aber vom Richter den Umständen des Falles angepasst werden. $^{98}$

d) Acción de cumplimiento

Nach Art. 200 Nr. 6 der Verfassung von 1993 kann die „Erfüllungsklage“ gegen jede Behörde oder jeden Amtsträger wegen Nichtbeachtung einer gesetzlichen Norm oder eines Verwaltungsakts erhoben werden. Dabei handelt es sich eher um ein verwaltungsrechtliches Verfahren vor den Zivilgerichten, das auf Art. 87 der kolumbianischen Verfassung von 1991 zurückgeht. ${ }^{99}$ Es ist näher in Art. 66-74 des Verfassungsprozessgesetzbuchs geregelt und gewinnt in der Praxis zunehmend an Bedeutung. ${ }^{100}$

Ein Schwerpunktheft der Rechtszeitschrift der katholischen Universität Lima (PUC) behandelt das Habeas-data-Verfahren in Spanien, USA, Brasilien und anderen lateinamerikanischen Ländern: Derecho 51 (1997) mit Beiträgen für Peru von Domingo García Belaunde und Francisco Eguiguren Praeli.

Geschützt nach Art. 200 Nr. 3 Verf. idF durch Gesetz 26470 sind die in Art. 2 Nr. 5 und 6 genannten informationellen Grundrechte, die deshalb vom Amparo-Verfahren ausdrücklich ausgenommen wurden. Der in Art. 2 Nr. 7 Verf. verbürgte Ehrenschutz sowie das Recht am eigenen Bild waren ursprünglich auch vom Habeas-data-Verfahren umfasst, fallen aber jetzt wieder unter das Amparo-Verfahren (Art. 37 Nr. 8 CPConst). Vgl. dazu noch Gorki Gonzales Mantilla, El hábeas data y el derecho a la propia imagen en la Constitución de 1993, Revista del Foro 81 (1993) Nr. 2 S. 143 ff.

Das Gesetz 26301 ist dadurch ausdrücklich aufgehoben; anders irrig Leny Palma Encalada, El proceso de hábeas data y de cumplimiento en el diseño del Código Procesal Constitucional, RevJurPerú 55 (2005) Nr. 62 S. 125 ff. (132, 136).

Schrifttum: Eloy Espinosa-Saldaña Barrera, El hábeas data en el nuevo Código Procesal Constitucional, RevPerJurispr 7 (2005), Compendio espezialido Nr. 40 S. 19 ff.; siehe auch die Rechtsprechungsübersichten ebd. S. 37-96 und in RevPerJurispr 7 (2005) Nr. 47 S. 244 ff., 8 (2006) Nr. 59 S. 445 ff.; Klagmuster: NormLeg 346 (2005) II 331 ff., 354 (2005) II 233 und 359 (2006) II 173.

Für einen Vergleich beider Regelungen siehe Ernesto Rey Cantor / María Carolina Rodríguez, La acción de cumplimiento en el Perú, NormLeg 280 (1999) A-94 ff.; vgl. auch Palma Encalada (Fn. 97), 135 ff.

100 Vgl. aus der neueren Rechtsprechung: Trib.Const. 17.5.2005, RevPerJurispr 8 (2006) Nr. 61 S. 223 (drohender Konkurs der Gemeinde kein Einwand gegen Zahlungsverpflichtung); 17.5.2005, ebd. Nr. 64 S. 210; 17.5.2005, ebd. Nr. 65 S. 259; 21.11.2005, ebd. Nr. 59 S. 294; 13.11.2006, ebd. Nr. 70 S. 233; 23.9.2005, NormLeg 360 (2006) II 217; 28.2.2006, ebd. 362 (2006) II 101 ff.; 19.7.2006, ebd. 363 (2006) II 142. Ein Klagmuster mit näheren Hinweisen ist abgedruckt in RevJurPerú 2007 Nr. 75 S. 20 ff. 


\section{Normenkontrollklagen}

In den Art. 75-108 enthält das Verfassungsprozessgesetzbuch eine ausführliche Regelung derjenigen Verfahren, die eine abstrakte Prüfung von Rechtsnormen auf ihre Verfassungsoder Gesetzmäßigkeit ermöglichen. Dies ist zum einen die Popularklage vor den ordentlichen Gerichten gegen untergesetzliche Normen, zum anderen die Klage vor dem Verfassungsgericht gegen alle Normen mit Gesetzesrang. ${ }^{101}$ Die einleitenden „Gemeinsamen Vorschriften“ (Art. 75-83) beziehen sich überwiegend nur auf eines der beiden Verfahren und werden deshalb nachfolgend im jeweiligen Zusammenhang behandelt.

a) Acción de inconstitucionalidad

Die „Klage auf Verfassungswidrigkeit“ von formellen Gesetzen und anderen Normen mit Gesetzesrang ist vor dem Verfassungsgericht zu erheben. Sie wurde erstmals durch die Verfassung von 1979 eingeführt (Art. 298 Nr. 1) ${ }^{102}$ und in die Verfassung von 1993 übernommen (Art. 200 Nr. 4). Bis dahin konnte die Verfassungswidrigkeit von Gesetzen nicht durch eine selbständige Klage geltend gemacht werden. ${ }^{103}$ Ihre nähere Regelung fand die ,acción de inconstitucionalidad“ zunächst im Gesetz 23385 von 1982, später unter Fujimori im Gesetz 26435 von 1995, das jedoch durch seine Quorums-Erfordernisse einen Erfolg der Klage praktisch ausschloss (oben C I). Zudem wurde durch das nachfolgende Gesetz 26618 die Klagefrist von sechs Jahren auf sechs Monate verkürzt. Nach dem Sturz Fujimoris wurden diese Beschränkungen durch die Gesetze 27780 und 27850 von 2002 aufgehoben. ${ }^{104}$ Seither hatte das Verfassungsgericht in zahlreichen Fällen über die Verfassungsmäßigkeit wichtiger gesetzlicher Regelungen zu befinden. ${ }^{105}$

Angefochten werden können nach Art. 200 Nr. 4 der Verfassung alle Normen mit Gesetzesrang, welche die Verfassung nach Form oder Inhalt verletzen. Dazu gehören nach dieser

101

Siehe dazu Víctor Julio Ortecho Villena, Control de normas y el Código Procesal Constitucional, RevJurPerú 54 (2004) Nr. 58 S. 13 ff.; Julio A. Fernández Cartagena / José Miguel Cárdenas Mares, La acción de inconstitucionalidad y la acción popular en el nuevo Código Procesal Civil, 102 NormLeg 340 (2004) II 21 ff.

Siehe zur historischen Entwicklung ausführlich Eloy Espinosa Saldaña B., El tratamiento del control de la constitucionalidad en el Perú: Balance y perspectivas, Prudentia Iuris (Buenos Aires) 31 (1993) $7 \mathrm{ff}$.

103 C.S. 30.12.1969, RevJurisprPer 29 (1970) 478; 11.5.1970, ebd. 1357.

104 Siehe oben Fn. 44.

105 Eine Übersicht über die Jahre 2002-2004 in NormLeg 346 (2005) II 520 ff. weist für diesen Zeitraum 47 in der gleichen Zeitschrift veröffentlichte Entscheidungen nach, von denen 19 zur Feststellung der Verfassungswidrigkeit gelangten. Seitdem hat die Zahl der Verfahren noch wesentlich zugenommen. $\mathrm{Zu}$ den einzelnen Fallgruppen in der Praxis der Gerichts und den verschiedenen Möglichkeiten der Entscheidung siehe Arelí Valencia Vargas, Reflexiones sobre la actuación del Tribunal Constitucional superando la visión tradicional de "legislador negativo", RevPerJurispr 7 (2005) Nr. 56 S. 43, 50 ff. 
Vorschrift neben den formellen Gesetzen ${ }^{106}$ die Gesetzgebungsdekrete und Notverordnungen, ${ }^{107}$ ferner die Staatsverträge, Geschäftsordnungsregeln des Kongresses, Regionale Normen von allgemeinem Charakter sowie kommunale Satzungen. ${ }^{108}$ Die Gesetzesqualität der beiden letzten ist zwar umstritten, die Zulässigkeit der Normenkontrolle vor dem Verfassungsgericht aber heute unzweifelhaft. ${ }^{109}$ Zur Erhebung der Klage sind nach Art. 203 der Verfassung von 1993 berechtigt: 1. der Präsident, ${ }^{110}$ 2. der Oberste Staatsanwalt (Fiscal de la Nación), 3. der Ombudsmann (Defensor del Pueblo), 4. ein Viertel der gesetzlichen Zahl der Parlamentsmitglieder (d.h. 30 von 120), 5. eine Anzahl von 5000 Bürgern (bei kommunalen Satzungen genügt auch ein Prozent der jeweils Wahlberechtigten ${ }^{111}$ ), 6. die Präsidenten der Regionen sowie die Provinzvorsteher in Angelegenheiten ihrer Zuständigkeit $^{112}$, 7. die Berufskörperschaften wie etwa die Anwaltskammern in Angelegenheiten ihres Fachgebiets.

Das Verfassungsprozessgesetzbuch von 2004 enthält jetzt in den Art. 75-83 und 98-108 eine ausführliche Regelung der „Acción de inconstitucionalidad“. Die Anfechtungsfrist beträgt danach für alle Normen grundsätzlich sechs Jahre, bei Staatsverträgen sechs Monate; eine konkrete Normenkontrolle bleibt auch nach Ablauf dieser Frist möglich

106

Einschließlich der verfassungsändernden Gesetze; näher dazu Marcial Rubio Correa, El Tribunal sostiene que todo acto y todo ejercicio de competencias en el derecho está sometido a control constitucional, NormLeg 351 (2005) II 85 ff.

107

Gesetzgebungsdekrete (Decretos Legislativos) sind Verordnungen mit Gesetzeskraft aufgrund ausdrücklicher gesetzlicher Ermächtigung (Art. 104, 125 Nr. 2 Verf.); Notverordnungen (Decretos de Urgencia) mit Gesetzeskraft kann der Präsident unter bestimmten Voraussetzungen aus eigenem Recht erlassen (Art. 118 Nr. 19 und Art. 74 Abs. 3 Verf.).

Auf regionaler Ebene werden „Normas regionales de carácter general“ von den „Consejos Regionales" beschlossen (Art. 191 Verf. idF. durch Gesetz 28607 von 2005). Auf der Ebene der Provinzen und Distrikte werden kommunale Satzungen (Ordenanzas Municipales) von den jeweiligen Kommunalvertretungen (Consejos Municipales) erlassen (Art. 194 Verf. idF. durch Gesetz 28607 von 2005).

109

Vgl. dazu Hugo Gómez Apac, El sistema legislativo peruano, RevJurPerú 55 (2005) Nr. 62 S. 255, 261 ff., 269 ff. Für die Kontrolle der „ordenanzas municipales“ durch das Verfassungsgericht siehe etwa Trib.Const.16.5.2005, NormLeg $351 \S 168$. Daneben ist in bestimmten Fällen die Amparo-Klage möglich, Trib.Const. 23.1.2000, NormLeg 305, A-6; siehe schon früher C.S. 17.9.1984, RevJurPerú 37 (1986) 410.

${ }^{110}$ Er bedarf dafür der Zustimmung des Ministerrats und wird im Prozess durch einen Minister vertreten (Art. 99 Abs. 1 CPConst). Auch verfassungsrechtliche Bedenken nachgeordneter Behörden können an den Ministerrat herangetragen werden und auf diese Weise das Verfahren in Gang setzen; D.S. 043-2005-PCM v. 15.6.2005, NormLeg 349 § 149 (geändert durch D.S. 060-2006PCM v. 21.9.2006, ebd. 364 § 153).

111 Das gilt nicht für regionale Normen; anders noch Art. 25 des Gesetzes 26435 von 1995 (siehe jetzt Art. 98 CPConst).

112

Sie bedürfen dafür der Zustimmung des Regionalen Koordinationsrates (bestehend aus den Provinzvorstehern) bzw. des jeweiligen Provinzrates. 
(Art. 100). Nach Zulassung der Klage ist das Verfahren unabhängig vom Prozessverhalten der Parteien auch von Amts wegen fortzuführen (Art. 106). Die der Klage stattgebende Entscheidung ist im Gesetzblatt zu verkünden und setzt die betreffende Norm mit dem folgenden Tag außer Kraft (Art. 81 Abs. 1). ${ }^{113}$ Zugleich werden die damit verbundenen oder darauf beruhenden Normen aufgehoben (Art. 78). Eine Rückwirkung ist gemäß Art. 204 der Verfassung grundsätzlich ausgeschlossen, eine Wiederaufnahme abgeschlossener Prozesse nur im Straf- oder Steuerrecht möglich (Art. 83). ${ }^{114}$ Bei steuerrechtlichen Vorschriften muss das Gericht die zeitlichen Auswirkungen der Entscheidung ausdrücklich festlegen (Art. 81 Abs. 2). Wird die Klage (außer aus formalen Gründen) abgewiesen, so ist diese Entscheidung für alle Gerichte bindend und schließt eine konkrete Normenkontrolle aus (Art. VI Abs. 3, Art. 82).

\section{b) Acción popular}

Das Rechtsinstitut der Popularklage war in Peru bereits in der Verfassung von 1933 vorgesehen (Art. 133), fand aber seine gesetzliche Grundlage erstmals im Gerichtsverfassungsgesetz von 1963 (Gesetz 14605, Art. 7). Verordnungen oder andere von der Verwaltung erlassene Normen von allgemeinem Charakter, welche gegen Verfassung oder Gesetz verstießen, konnten danach vor den ordentlichen Gerichten in einem auf reine Rechtsfragen beschränkten Verfahren angefochten werden. In dieser Form wurde die Popularklage ebenso in die Verfassung von 1979 (Art. 295 Abs. 4) wie in die Verfassung von 1993 (Art. 200 Nr. 5) übernommen, wo sie nunmehr zu den Verfassungsklagen gezählt wird. Eine eigenständige Regelung erhielt sie durch das Gesetz 24968 von 1988, das heute durch die entsprechenden Vorschriften des Verfassungsprozessgesetzbuchs von 2004 abgelöst ist. $^{115}$

Nach Art. 76 dieses Gesetzbuchs ist eine Popularklage zulässig gegen Verordnungen, Verwaltungsvorschriften oder Erlasse von allgemeinem Charakter, welche die Verfassung oder das Gesetz verletzen oder die nicht in der durch Verfassung bzw. Gesetz vorgeschriebenen Form zustande gekommen oder verkündet worden sind. ${ }^{116}$ Unzulässig ist die Klage nach der Rechtsprechung, wenn es sich bei den angegriffenen Normen um reine Ausführungsbestimmungen ohne eigenen Regelungscharakter oder interne Satzungen ohne

Das entspricht Art. 204 Verf. 1993. Dagegen musste das Gericht nach Art. 301 der Verfassung von 1979 bei formellen Gesetzen dem Kongress 45 Tage Zeit zur Aufhebung geben; zur Problematik siehe Saligmann (Fn. 32), 140.

Diese Ausnahmen stützen sich auf Art. 74 Abs. 4 und Art. 103 Satz 2 der Verfassung.

15 Schrifttum: José Paulo Césare Sifuentes, El proceso de acción popular, NormLeg 348 (2005) II 187 ff., und die Autoren in Fn. 101.

116

Eine inhaltliche Kontrolle der Norm vor ihrer Verkündung, wie sie Art. 5 des Gesetzes 24968 von 1988 zuließ, ist im Verfassungsprozessgesetzbuch dagegen nicht mehr vorgesehen. 
Außenwirkung handelt. ${ }^{117}$ Die Popularklage kann von jedermann ohne weitere Legitimation erhoben werden (Art. 84); daneben wird von der Rechtsprechung auch die AmparoKlage zugelassen, wenn der Kläger durch die angegriffene Regelung unmittelbar in seinen verfassungsmäßigen Rechten betroffen ist. ${ }^{118}$ In der Praxis hat die Popularklage deshalb bisher keine größere Bedeutung erlangt. ${ }^{119}$ Zuständig für die Popularklage ist grundsätzlich das Obergericht (Corte Superior) in Lima, bei regionalen oder lokalen Normen das örtlich entsprechende Obergericht (Art. 85). Nähere Vorschriften zum Verfahren enthalten die Art. 86-97 des Verfassungsprozessgesetzbuchs; die Frist zur Erhebung der Klage beträgt fünf Jahre seit der Verkündung der betreffenden Norm (Art. 87). Das der Klage stattgebende Urteil hat allgemeine Wirkung (erga omnes), über eine mögliche Rückwirkung befindet das Gericht nach seinem Ermessen (Art. 81 Abs. 3). In zweiter und letzter Instanz entscheidet der Verfassungssenat des Obersten Gerichtshofs; bis zu seiner Entscheidung kann die Wirkung der angefochtenen Norm suspendiert werden (Art. 93-95).

\section{Kompetenzkonflikte}

Die sog. Kompetenzkonflikte wurden dem Verfassungsgericht durch Art. 202 Nr. 3 der Verfassung von 1993 neu zugewiesen und in Art. 109-113 des Verfassungsprozessgesetzbuchs näher geregelt. Es handelt sich um Organstreitigkeiten über die in Verfassung und Gesetz festgelegten Zuständigkeiten und Befugnisse, an denen die Zentralregierung, die Regional- und Munizipalregierungen und andere Staatsorgane beteiligt sein können. ${ }^{120}$ Von dieser Möglichkeit wird zunehmend Gebrauch gemacht. ${ }^{121}$

C.S. 13.12.1999, NormLeg 288, A-38: 22.5.2002, RevPerJurispr 5 (2003) Nr. 33 S. 19.

118 C.S. 30.9.1994, NormLeg 253, A-21 (Decreto Supremo); 12.8.1992, ebd. 246, J-9 (Resolución Suprema); Trib.Const. 8.7.1998, ebd. 278, A-1 (Resolución Ministerial); 10.5.2004, RevPerJurispr 6 (2004) Nr. 45 S. 33 (Resolución Directoral Regional); 16.10.2001, ebd. 4 (2002) Nr. 11 S. 13 (Resolución de Alcaldía); 23.10.1998, NormLeg 274, A-10 (Edicto Municipal); 27.2. und 22.3.2001, ebd. 301, A-32f. (Resolución de Gerencia); weitere Entscheidungen anderer Gerichte ebd. 261, A-1 und 273, A-21.

119 Vgl. die Motive zum Verfassungsprozessgesetzbuch bei Fernández Cartagena / Cárdenas Mares (Fn. 101), 25. Beispiel für eine Popularklage aus neuerer Zeit in NormLeg 262 (2006) II 113 (Decreto Supremo).

120

Siehe dazu Ortecho Villena (Fn. 101), 23 ff. und den Überblick in NormLeg 349 (2005) II 197 f. Auch für diese Klagen gilt das genannte D.S. 043-2005-PCM (Fn. 110). Die noch in Art. 49 des Gesetzes 26435 von 1995 vorgesehene Beteiligung von Privatpersonen wurde in das Verfassungsprozessgesetzbuch nicht übernommen.

Auf der homepage des Gerichts sind für die Zeit von 1995-2000 nur 10, für die folgenden Jahre 43 Verfahren ausgewiesen. Zu den Wirkungen einer solchen Entscheidung siehe Trib.Const. 13.2.2007, RevJurPerú 2007 Nr. 74 S. 23. 


\section{Internationale Gerichtsbarkeit}

Nach Erschöpfung des internen Rechtsweges kann die Verletzung verfassungsmäßiger Rechte weiter vor den internationalen Gerichten verfolgt werden, denen Peru sich vertraglich unterworfen hat; dies ist auch in der Verfassung deklaratorisch festgeschrieben. ${ }^{12}$ Unter der Regierung Fujimori widerrief Peru - während des laufenden Verfahrens im Fall der abgesetzten Verfassungsrichter - seine Unterwerfung unter die Zuständigkeit des Interamerikanischen Gerichtshofs für Menschenrechte, was dieser aber nicht akzeptierte. ${ }^{123}$ Nach dem Sturz Fujimoris wurde der Widerruf zurückgezogen ${ }^{124}$ und die Erfüllung von Entscheidungen internationaler Gerichte durch Gesetz 27775 von 2002 näher geregelt. ${ }^{125}$ Ergänzende Vorschriften enthält dazu heute das Verfassungsprozessgesetzbuch von 2004 in seinen Art. 114-116 über die Mitwirkungspflichten der staatlichen Organe. Die in Verfahren gegen Peru ergangenen Entscheidungen werden seither im peruanischen Gesetzblatt veröffentlicht. $^{126}$

\section{Ausblick}

Die peruanische Verfassung von 1993, die unter einem diktatorischen Regime auf zweifelhafte Weise zustande gekommen war, hat unter demokratischen Verhältnissen durch Anerkennung in der politischen Praxis ihre Legitimation erlangt. Auf dieser Grundlage wurde auch die Verfassungsgerichtsbarkeit neu geregelt. Seit der Jahrtausendwende konnte das

Der Widerruf erfolgte durch Resolución Legislativa 27152 v. 8.7.1999. Die Entscheidung des Gerichtshofs erging am 24.9.1999, abgedruckt in La Ley (Buenos Aires) 2000-B, 72. Siehe dazu Juan Carlos Hitters, Imposibilidad de retirarse de la jurisdicción de la Corte Interamericana (El caso de Perú), ebd. 1999-F, 893 ff.; Douglass Cassel, Peru withdraws from the Court, Human Rights Law Journal 20 (1999) 167 ff.

Siehe Resolución Legislativa 27401 v. 18.1.2001.

Zur Durchführung siehe D.S. 006-2006-JUS v. 17.2.2006, NormLeg $357 \S 161$. Ein neuerer Gesetzentwurf 853/2006-CR v. 10.1.2007 fordert die Aufhebung des Gesetzes und die Gleich126 stellung der internationalen mit den nationalen Entscheidungen.

So die Entscheidungen des Interamerikanischen Gerichtshofs vom 14.3.2001 (Barrios Altos), El Peruano v. 8.4.2005 (NormLeg $347 \S$ 027), dazu in Peru Trib.Const. 29.11.2005, RevPerJurispr 8 (2006) Nr. 60 S. 186; 8.7.2004 (Hermanos Gómez Paquiyauri), El Peruano v. 3.9.2005 (NormLeg $352 \S 009$ ); 25.11 .2004 (Berenson Mejía), El Peruano v. 20.8.2005 (NormLeg $351 \S 199$ ); 3.3.2005 (Huilca Tecse), El Peruano v. 20.8.2005 (NormLeg 351 § 196); 25.11.2005 (Gómez Palomino), El Peruano v. 12.2.2006 (NormLeg 357 § 109); 25.11.2005 (García Asto/Ramírez Rojas), El Peruano v. 5.3.2006 (NormLeg 358 § 054); 7.2.2006 (Acevedo Jaramillo), El Peruano v. 9.6.2006 (NormLeg $361 \S 098$ und 377 § 196); 6.4.2006 (Baldeón García), El Peruano v. 30.5.2007 (NormLeg 377, I-10); 29.11.2006 (La Cantuta), El Peruano v. 23.6.2007 (NormLeg $379 \S 249)$. 
Verfassungsgericht wieder aktiv seine Rolle als Hüter der Verfassung wahrnehmen. ${ }^{127}$ Dies hat zu einer erheblichen Zunahme der Verfahren geführt, was die Funktionsfähigkeit des Gerichts beeinträchtigen kann ${ }^{128}$. Vor allem im Bereich der Rechtsschutzklagen ist in den letzten Jahren die Zahl der jährlichen Eingänge etwa um das Zehnfache auf mehr als 10000 gestiegen; das Gericht sah sich deshalb schließlich genötigt, einem seiner beiden Senate die Vorprüfung dieser Verfahren zu übertragen und zugleich die Kriterien der Zulassung zu verschärfen. ${ }^{129}$ Weitergehend ist in einem neueren Gesetzentwurf vorgesehen, dem Gericht selbst als letzter Instanz bei den Rechtsschutzklagen die Auswahl der verfassungsrechtlich relevanten Verfahren nach eigenem Ermessen zu überlassen. ${ }^{130}$ Mit seiner aktiven Rolle hat sich das Verfassungsgericht aber auch Kritik zugezogen, die ihm insbesondere bei der abstrakten Normenkontrolle eine unzulässige Überschreitung seiner Funktionen vorwirft. So zielt ein anderer Gesetzentwurf darauf ab, die Kompetenzen des Verfassungsgerichts generell einzuschränken. ${ }^{131}$ Wenngleich diese Forderung überzogen erscheint, so können einzelne Entscheidungen des Verfassungsgerichts, das auch in seiner jetzigen Besetzung nicht frei von politischen Einflüssen ist, durchaus kritisch gesehen werden. ${ }^{132}$ Insgesamt zeichnet sich jedoch eine positive Entwicklung ab, die noch vor einem Jahrzehnt so nicht erwartet werden konnte.

127 In einem Leitartikel der vom Gericht herausgegebenen „Gaceta del Tribunal Constitucional“ (No. 5, enero-marzo 2007) wird dies auf drei Gründe zurückgeführt: 1) die allgemeine demokratische Entwicklung, 2) die Erneuerung des Gerichts durch Ernennung neuer Verfassungsrichter, 3) die gelungene Integration des Gerichts in das politische System.

Das Gericht hat deshalb jetzt Grundsätze aufgestellt, nach denen Anwälte bei missbräuchlichen Klagen mit einer Strafgebühr belegt werden können: Trib.Const. 29.8.2005, NormLeg 365 II 189.

Änderung des Reglamento Normativo durch Resolución Administrativa 31-2006-P/TC v. 1.3.2006, NormLeg 358 § 019. Kritisch dazu Eugenia Ariano Deho, La "superlativa" protección de los derechos fundamentales del Tribunal Constitucional y su nueva (e inconstitucional) "chambres de requêtes", RevPerJurispr 8 (2006) Nr. 70 S. 3 ff., dort auch genauere Angaben zur Rechtsmittelstatistik (auf S. 5).

131 Gesetzentwurf 14321/2005-CR v. 20.1.2006.

132 Siehe dazu Aníbal Quiroga León, Los excesos del Tribunal Constitucional Peruano: A propósito del control concentrado de la Constitución, NormLeg 353 (2005) II 145 ff.; Leny Palma Encalada, Tribunal Constitucional vs Poder Legislativo, Comentarios a través de la Jurisprudencia, RevPerJurispr 8 (2006) Nr. 65 S. 47 ff.; Daniel E. Cerna Salazar, Pero ¿y el Tribunal Constitucional? ... acaso se trata de ¿"la isla bonita"?, ebd. Nr. 67 S. 346 ff.; Luis Castillo Córdova, ¿Activismo extralimitado del Tribunal Constitucional?, RevJurPerú 2007 Nr. 77 S. 19 ff. 